Diánoia, vol. 22, no. 22, 1976

\title{
RELATIVISMO Y CIENCIAS SOCIALES
}

I

En las discusiones corrientes acerca de la naturaleza de las ciencias sociales ocupan un lugar de preeminencia cuestiones tales como la "carga" valorativa de los fenómenos que son objeto de investigación, el compromiso valorativo de los investigadores sociales, el condicionamiento valorativo de los marcos teóricos, de los conceptos y de los términos utilizados por los científicos sociales, etcétera. Estas cuestiones surgen -entre muchas otras - al enfrentarse dos puntos de vista contrapuestos: el que atribuye a las ciencias sociales un carácter sui generis que permite distinguirlas claramente de las ciencias naturales y el que ubica a las ciencias sociales en un continuo con las ciencias naturales, continuo en el que éstas juegan el papel de paradigma. Es difícil minimizar el interés y la importancia de esta polémica, aunque cabe reconocer, sin embargo, serias dificultades en las maneras en que se la suele plantear. Una de tales dificultades surge, precisamente, en relación con el ámbito valorativo y consiste en la propensión generalizada de los filósofos de la ciencia a discutir los modos en que puede o no manifestarse el compromiso valorativo en las ciencias sociales, ignorando las contribuciones efectuadas en el ámbito de la filosofía moral; es decir, ignorando los aportes efectuados en el campo propio en que tales problemas se analizan especificamente. Así, se discute a veces acerca de la "objetividad" o de la falta de "objetividad" de los juicios valorativos, pasando por alto las elucidaciones de indole semántica, ontológica y gnoseológica efectuadas en relación con dicho tema por los filósofos de la moral. A veces se discute acerca del carácter valorativo o no valorativo (descriptivo) de los términos cruciales empleados en sus teorías por los científicos sociales, pero no se especifican criterios mínimamente elaborados que permitan distinguir descripción de valoración ni se fijan posiciones acerca de la posible reducción de los términos y juicios valorativos a términos y juicios descriptivos, por ejemplo. A estos temas los filósofos de la moral han hecho también importantísimos aportes. Otras veces se polemiza sobre los supuestos relativistas que trae aparejados —según se supone - el reconocimiento de compromisos valorativos, pero no se presta atención a las distinciones conceptuales y metodológicas que han propuesto al respecto los filósofos de la moral, ni las consecuencias teóricas que trae aparejado adoptar una posición relativista. Los ejemplos de este tipo pueden multiplicarse. Adviértase que la crítica a esta manera de discutir problemas valorativos no se funda en la mera alegación de supuestas prioridades teóricas. El planteo apunta a algo más profundo. Si, como creo, toda buena dis- 
cusión filosófica procura alcanzar niveles cada vez más elaborados de comprensión conceptual y de construcción tébica, es obvio que la polémica acerca del compromiso valorativo de las ciencias sociales sólo alcanzará niveles óptimos, en el sentido indicado, en tanto tome en cuenta aportes efectuados en el área a la que los problemas valorativos pertenecen por derecho propio, es decir, a la filosofía de la moral. Está claro que la incorporación de tales aportes no solucionará automáticamente la cuestión de fondo, pero no cabe duda de que enriquecerá de modo adecuado su contenido.

En el presente trabajo me propongo analizar algunas cuestiones que plantea el relativismo. La importancia que reviste este tema permite omitir mayores comentarios acerca de su elección. Interesa aclarar, en cambio, que no me propongo exponer el contenido de las posiciones sustentadas al respecto por diferentes filósofos de la moral, sino elaborar un enfoque global que muestre, por un lado, la necesidad de formular determinadas distinciones metodológicas y conceptuales, y que por el otro lado, siente las bases que a mi criterio permiten juzgar acerca de la viabilidad de distintas variantes de relativismo. Este análisis ocupa las secciones 1 a 4 . En la sección 5 me detendré a considerar, brevemente, algunas consecuencias del relativismo generalmente asociado a la alegación de un compromiso valorativo en las ciencias sociales.

\section{Niveles metodológicos y variantes relativistas}

Un requisito necesario para ubicar adecuadamente la discusión acerca del relativismo, consiste en distinguir, a grandes rasgos, los distintos niveles en que se pueden ubicar los tipos de reflexión sobre la moralidad. Digo que es un requisito necesario porque una de las primeras dificultades con que se tropieza al encarar el tema del relativismo tiene origen en la existencia de diferentes variantes de posiciones relativistas. $Y$ es bastante claro que una de las maneras de comenzar a ordenarlas es a través de su ubicación en niveles distintos, de acuerdo a criterios explícitos. Considero que la distinción de niveles metodológicos que propondré es bastante buena. Entre otras razones, porque se adecúa a las modalidades teóricas practicadas efectivamente. Soy .consciente, sin embargo, de algunas dificultades que plantea y, en definitiva, de su carácter instrumental. Pero dado el hecho -que considero innegable - de que alguna distinción de niveles debe trazarse, la razón expuesta es suficiente para decidirme al respecto. El planteo puede efectuarse en los siguientes pasos.

Primero, distinguiré entre la moralidad (un fenómeno social/individual sumamente complejo) y el estudio de la moralidad.

Segundo, dentro del ámbito de la reflexión teórica distinguiré (a) el estudio teórico-empirico de la moralidad, que abarca problemas relativos a las opiniones y actitudes morales de individuos y/o de grupos sociales, y/o 
al origen, evolución y desarrollo de tales opiniones y actitudes, etcétera; (b) el estudio normativo de la moralidad, que abarca problemas relativos a la obligación moral, al (a los) bien(es) intrínseco(s), a los valores y virtudes morales, al (a los) ideal(es) de vida, etcétera, y (c) el estudio teórico-conceptual de la moralidad, que abarca problemas relativos al significado de las palabras usadas en contextos típicamente morales, al contenido de los conceptos respectivo, a la naturaleza y status de aquello a lo que tales palabras se "refieren" o a lo que tales conceptos mientan, a la elucidación de los elementos del esquema conceptual propio de la moralidad, etcétera. Este esquema mueve a distinguir a (a), por un lado, y a (b) y (c), por el otro, lo cual supone la clásica distinción entre investigación científica e investigación filosófica. Además, (b) presenta una curiosa atipicidad debida, sin duda, a los rasgos sui generis de la moralidad. Esa atipicidad genera "tensiones" en cuanto a la relación con (c); por ejemplo, acerca de su eventual neutralidad, acerca de la pertinencia de (b) como actividad filosófica, etcétera. No me ocuparé aquí de estas y de otras cuestiones que surgen en relación con el esquema propuesto. (Cf., al respecto Brandt [1959], Hare [1960] y Rabossi [1971]).

Tercero, concordantemente con la distinción de niveles trazada distinguiré tres tipos de relativismo: (a) relativismo socio-cultural, (b) relativismo normativo y (c) relativismo metaético (o relativismo ético, en sentido estricto). Cada uno de estos tipos ofrece diferentes versiones especificas. Por otra parte, aś como son distintos los criterios generales de adecuación, el ámbito de validez y las condiciones de validación en cada uno de los niveles metodologicos señalados, también son distintos, concordantemente, cada uno de los tipos de relativismo que he distinguido.

En base al planteo así esquematizado se puede encarar el análisis de los tipos de relativismo especificados. Tal tarea será desarrollada en las tres secciones siguientes.

\section{Relativismo socio-cultural}

El denominado "relativismo socio-cultural" (en adelante $R_{1}$ ), llamado corrientemente "relativismo cultural", es una tesis de carácter empírico fundada en investigaciones de antropólogos, sociólogos y psicólogos y aceptada, expresa o implicitamente, por muchos de ellos. En términos generales, $\mathbf{R}_{\mathbf{x}}$ registra el hecho de que grupos sociales distintos difieren en sus pautas morales y explica tal circunstancia en base a las diferencias en el condicionamiento socio-cultural. Más estrictamente, $R_{1}$ se puede expresar de la siguiente manera:

$T_{1}$ : En grupos sociales distintos, digamos $G_{1}$ y $G_{2}$, un mismo tipo de acto al que se atribuye contenido moral puede ser juzgado, en general y en un gran número de casos, de manera diferente; 
$T_{2}:$ El comportamiento moral y las valoraciones asociadas a él están condicionados socio-culturalmente, y las diferencias señaladas en $T_{1}$ se explican porque los miembros de $G_{1}$ y de $G_{2}$ pertenecen a medios culturales distintos;

Corolario: Dada la situación descripta en $T_{1}$, un mismo tipo de acto al que se atribuye contenido moral será, por ejemplo, bueno, correcto, encomiable para los miembros de $G_{1}$ e incorrecto, malo, censurable para los miembros de $\mathbf{G}_{2}$.

Conviene recordar que $R_{1}$ posee carácter empírico y que, en tanto tal, depende en cuanto a su aceptabilidad del grado de corroboración o de refutabilidad que pueda otorgársele. Claro que una decisión al respecto es difícil de adoptar. Es obvio que un antropólogo preocupado por encontrar rasgos diferenciales en los distintos grupos sociales que estudia, acopiará datos que verifican $R_{1}$, mientras que otro antropólogo que dé cabida en su esquema teórico a la posibilidad de que existan universales éticos, por ejemplo, será renuente a reconocer la relevancia de tales datos o los interpretará de manera distinta. Esta observación no pretende sugerir que resulte imposible determinar el grado de aceptabilidad de $R_{1}$. Sólo se propone advertir que en el caso de $R_{1}$ no es fácil especificar el grado de corroboración o de refutación pues existen de hecho condiciones previas a la corroboración de $\mathbf{R}_{\mathbf{1}}$-por ejemplo, el interés en especificar rasgos diferenciales o, por el contrario, el interés en desarrollar enfoques unificadores- que determinan, en parte al menos, la manera de seleccionar y de evaluar el material empírico correspondiente.

$\mathrm{R}_{1}$ presenta, además, otro tipo de dificultades teóricas. Me refiero al significado de ciertas expresiones cruciales en su formulación. Tomemos, por ejemplo, la expresión "el mismo tipo de acto" (o, "el mismo acto"). ¿Cuándo un acto es el mismo (o cuándo se trata del mismo tipo de acto) en dos grupos sociales diferentes? El trillado ejemplo del parricidio puede servir de ilustración. (Recuérdese que mientras que en nuestra sociedad el parricidio es condenado moralmente como el crimen más horrible, en otros grupos sociales, por ejemplo en algunas tribus esquimales, la muerte del padre por parte de los hijos es aceptada y, aún, estimulada, dadas las circunstancias). Prima facie puede decirse que el parricidio proporciona un ejemplo claro de un caso en el que el mismo tipo de acto, matar al padre, es objeto de valoraciones opuestas en grupos sociales distintos. Pero cuando un esquimal, por ejemplo, lleva a cabo actos que tienen por objeto hacer que su padre anciano se hunda en el. hielo y muera, y cuando un miembro de nuestra sociedad realiza u omite acciones que producen, también intencionalmente, la muerte de su padre, ¿en qué sentido puede afirmarse que ambos llevan a cabo el mismo acto? ¿Son acaso las mismas las razones que gulan a uno y a otro? ¿'Tienen los autores los mismos resultados in mente? ¿En base a qué criterios 
se afirma que en los grupos sociales en cuestión los actos correspondientes se juzgan apelando a principios morales contrapuestos? Puede argumentarse que si bien se puede decir, en cierto sentido, que ambos sujetos realizan el mismo acto (es decir, el acto que se describe con la expresión "provocar intencionalmente la muerte de un ser humano que es su progenitor"), en otro sentido -que es el que interesa moralmente- llevan a cabo actos diferentes: el esquimal lleva a cabo un acto misericordioso, una especie de eutanasia socialmente regulada, mientras que nuestro vecino, motivado por pasiones, instintos y propósitos innobles realiza un acto inmoral. Con otras palabras, el acto es el mismo desde un punto de vista físico y "biológico", pero no lo es desde un punto de vista psicológico o social. Estos últimos aspectos resultan fundamentales por ser aspectos en función de los cuales queda determinada la valoración correspondiente. De esto se sigue que, en el ejemplo dado, no es exacto que en los dos grupos sociales se realiza un mismo acto juzgado de distinta manera y que haya, en consecuencia, desacuerdo de fondo (desacuerdo último) en las respectivas valoraciones. Se supone que aclaradas las cuestiones de hecho relevantes, esquimales y no esquimales concordamos en que en nuestros grupos sociales rige un principio moral similar, por ejemplo, que es obligación de los hijos adultos velar por el bienestar de los padres ancianos, aliviando su sufrimiento, etcétera. Este planteo se puede generalizar, de modo que valga para todos los casos en los que se postulan diferencias en las valoraciones de "el mismo acto". Se supone que en todos ellos se arribará a conclusiones semejantes a las señaladas más arriba. Conviene aclarar en este punto el peso del argumento. No creo que de él se siga que $\mathbf{R}_{\mathbf{1}}$ es falsa o que se fundamenta en premisas falsas. (En contra, Asch [1962], caps. 11-14). En cambio, se sigue del argumento que $R_{1}$ incluye expresiones cuyo significado exige una aclaración adecuada. De no proporcionarse tal aclaración se sigue entonces del argumento que es posible invalidar $\mathbf{R}_{\mathbf{1}}$ en un plano conceptual, es decir, que es posible mostrar que ciertas expresiones cruciales de $R_{1}$ no están sujetas a las condiciones de uso que la tesis presupone acríticamente. Argumentaciones de un carácter parecido pueden efectuarse en relación con la traducibilidad de las expresiones usadas típicamente en contextos morales por grupos sociales que hablan lenguajes diferentes y con la indole precisa del condicionamiento del que habla $\mathbf{R}_{\mathbf{1}}$.

Supóngase que $R_{1}$ se formula de modo tal que se superan los problemas apuntados. Supóngase, además, que se reconoce fundadamente a $R_{1}$ un grado de corroboración satisfactorio. ¿Qué consecuencias prácticas y teóricas se siguen de ello? Restringiré la respuesta a tres puntos que poseen un interés peculiar para la cuestión de fondo, a saber, la validez de ciertos resultados prácticos que se suelen asociar a la aceptación de $\mathbf{R}_{1}$, la relevancia de $\mathbf{R}_{\mathbf{1}}$ para resolver la polémica filosófica entre absolutistas y relativistas y, finalmente, la conexión posible entre $R_{1}$ y ciertas teorias metaéticas. 
Basándose en el corolario que resulta de $T_{1}$ y $T_{2}$ y en la aseveración de que las valoraciones contrapuestas que se formulan en $G_{1}$ y $G_{2}$ son igualmente válidas - una aseveración indebidamente agregada en este nivel metodológico por cuanto no es parte legítima de $R_{1}$ ni se sigue de $R_{1}-$, se sostiene a veces que la aceptación de $R_{1}$ tiene como resultado la adopción de una actitud tolerante respecto de las opiniones, pautas y valoraciones morales distintas de las propias, o distintas de las del propio grupo. La tolerancia seria, pues, una secuela importante y atractiva asociada al relativismo sociocultural. Este planteo es, sin embargo, criticable. Si lo que se sostiene es que de hecho $\mathrm{R}_{1}$ ayuda a no ser intolerable, el planteo pierde interés: es posible que tal sea el caso, como es posible que $R_{1}$ no genere tolerancia o, aún, que sea la base para elaborar una actitud etnocéntrica. A su vez, si al afirmar que $\mathbf{R}_{1}$ favorece la tolerancia se pretende sostener que tal valor, la tolerancia, es encomiable o que es el valor digno de encomio, entonces el planteo tiene pretensiones exageradas que resulta dudoso justificar con la única ayuda de $R_{1}$ y de la igualdad de validez de juicios morales contrapuestos. Adviértase que una argumentación parecida puede hacerse respecto de un planteo paralelo a éste, esto es, el planteo sumamente corriente que consiste en extraer de $R_{1}$ más la aseveración indebida acerca de la igualdad de validez, consecuencias de tipo escéptico. Este es, por cierto, el planteo más corriente. Sin embargo, cabe afirmar que no se sigue en modo alguno de $R_{1}$. Y si se lo deriva de $\mathbf{R}_{1}$ y de la aseveración mencionada cabe argumentar que o bien se trata de una consecuencia que acompaña de hecho a $R_{1}$, en cuyo caso el planteo pierde interés teórico, o bien se presupone no ya la aseveración mencionada sino también otra aseveración también indebidamente agregada en este nivel metodológico, a saber, que la aceptación de que existen valoraciones contrapuestas igualmente válidas excluye la posibilidad de que existan métodos racionales para zanjar las controversias éticas. Como veremos en IV, ésta es una tesis filosófica que, obviamente, no tiene cabida en el nivel metodológico al que pertenece el relativismo socio-cultural.

En cuanto a la relevancia de $\mathbf{R}_{\mathbf{1}}$ para resolver la polémica filosófica entre relativistas y absolutistas corresponde formular, también, algunas observaciones de interés. Puede pensarse que si $R_{1}$ se encuentra suficientemente corroborada se sigue, en principio, la falsificación de toda postura absolutista, esto es, que la viabilidad del relativismo socio-cultural implica el rechazo de cualquier tesis filosófica de indole absolutista. Las cosas no son, sin embargo, tan sencillas, pues $\mathrm{R}_{1}$ no es ex hypothesi una tesis filosófica sino empírica y, además, la eventual relevancia filosófica de $R_{1}$ es materia de controversia. Que ello es așí se prueba en base al hecho de que todo filósofo que sostiene una posición absolutista tiene en cuenta la diversidad de sistemas y de valoraciones morales y que la posición que propone pretende dar cuenta, entre otras cosas, de tal circunstancia. La estrategia que generalmente se sigue al 
respecto es conocida: o bien se hace hincapié en las limitaciones cognoscitivas de los seres humanos, que impiden acceder plenamente al ámbito de los principios verdaderos y absolutos, de los valores últimos, etcétera, o bien se insiste en que la diversidad apuntada es sólo superficial ya que existe, en el fondo, un contenido común a todos los sistemas morales que sirve de fundamento a la corrección, veracidad, racionalidad, etcétera, de los juicios morales. No pretendo sugerir que estas maneras de argumentar están exentas de críticas. Creo que, en realidad, merecen ser criticadas, pero sólo a nivel filosófico. En suma, $\mathrm{R}_{\mathbf{1}}$ no refuta el absolutismo $\mathrm{y}$, consiguientemente, no prueba la corrección del relativismo, en tanto posiciones filosoficas. Con otras palabras, $\mathbf{R}_{\mathbf{1}}$ no es suficiente para probar el relativismo a nivel filosofico, aunque para ser relativista a ese nivel es necesario probar la viabilidad de $R_{1}$.

Por último, a partir de $R_{1}$ se puede pretender inferir ciertos rasgos propios de lo moral; por ejemplo, a partir de la diversidad que manifiestan las valoraciones morales puede afirmarse que la esfera emotiva tiene, de alguna manera, una conexión esencial con lo moral. Nuevamente corresponde advertir que tal cosa no se sigue de $\mathbf{R}_{1}$, lo cual no implica negar el importante papel que la esfera emotiva juega en el ámbito de la moral. Con otras palabras, $R_{1}$ no implica a nivel metático una posición emotivista (en realidad, $\boldsymbol{R}_{2}$ no implica en ese nivel ninguna posición), aunque puede señalarse que el emotivismo, como doctrina metaética, tiene consecuencias relativistas en el ámbito metaético al cual pertenece.

Estos comentarios acerca del relativismo socio-cultural pretenden enfatizar el carácter empírico de ese tipo de tesis relativista y, al mismo tiempo, llamar la atención acerca de algunas consecuencias que siguen de tal carácter y que corrientemente suelen ser pasadas por alto. Lo argumentado hasta aqui no se propone negar la aceptabilidad de $\mathbf{R}_{1}$. De lo que se trata en realidad es de trazar los límites de su validez efectiva.

\section{Relativismo normativo}

Cuando se pasa del nivel propio de la ética descriptiva al de la ética normativa se encuentra un tipo distinto de relativismo, denominado "relativismo normativo" o "relativismo moral" (en adelante $R_{2}$ ). $R_{1}$ no implica $R_{2}$. Una tesis como $\mathbf{R}_{1}$ que tiene como finalidad la descripción de cierto tipo de fenómenos no puede implicar, estrictamente, pautas acerca de cómo actuar, de cuál es el comportamiento correcto, etcétera. Esta función es la que pretende cumplir $\mathbf{R}_{\mathbf{2}}$.

No es fácil exponer el contenido de $R_{2}$ porque su formulación no es frecuente en contextos teóricos. Pero la siguiente versión se adecúa, creo, a sus rasgos fundamentales. Para un defensor de $R_{2}$, el principio fundamental que permite decidir qué debe hacerse, desde un punto de vista moral, es el 
siguiente: en una situación determinada un agente moral debe hacer lo que piensa que debe hacer; si el agente moral piensa que debe hacer $A$, entonces es correcto para ese agente moral hacer $A$. En términos generales, es correcto hacer lo que se piensa que se debe hacer; es incorrecto hacer lo que no se piensa que se debe hacer. Adviértase que $R_{2}$ no afirma una regularidad entre lo que los agentes morales piensan de hecho acerca de la corrección de los actos a realizar y la corrección efectiva de dichos actos. $\boldsymbol{R}_{2}$ tampoco enuncia un principio en favor de la "autenticidad" en materia moral; no estipula que sólo debemos realizar aquellos que realmente pensemos que es lo correcto hacer. Tampoco afirma $\mathbf{R}_{2}$ el principio alternativo de que un agente moral no debe ser censurado por hacer $A$ cuando realmente piensa que hacer tal cosa es para él correcto. $R_{2}$ postula que pensar que es correcto hacer un acto determinado es una condición suficiente para la corrección de dicho acto y que, en consecuencia, debemos hacer lo que pensamos que es correcto hacer.

$\mathbf{R}_{2}$ está sujeta a críticas de peso. En primer lugar, no da cabida a una actividad esencialmente asociada a la moralidad: la critica (censura) y/o la aprobación (encomio) de los actos realizados por agentes morales. Si "correcto" ("incorrecto") sólo es susceptible de calificar un acto de un agente moral cuando dicho agente moral piensa que el acto, digamos $A$, es correcto, entonces otro agente no podrá calificar al acto (con pretensiones críticas y en el mismo sentido que el agente actor) de incorrecto (correcto). La razón es que según $\mathrm{R}_{2}$ la corrección de $A$ supone que el agente actor piensa que $A$ es correcto. Por cierto que el segundo agente moral puede cuestionar cómo es que el agente actor ha llegado a pensar que $A$ era correcto 0 , aún, que no piensa en realidad que $A$ sea correcto. Pero ninguna de estas dos cosas puede asimilarse a criticar a un agente moral por haber hecho algo incorrecto. En segundo lugar, $\mathbf{R}_{2}$ elimina la posibilidad de discutir cuestiones de indole moral, una actividad que también está esencialmente asociada a la moralidad. $S i$ un agente piensa que $A$ es correcto y otro agente piensa que es incorrecto, normalmente diremos que ambos agentes difieren acerca de la corrección de $A$. Pero como la corrección es función de lo que piensa el primer agente moral, y la incorrección de $A$ es también función de lo que piensa el segundo agente moral, no hay posibilidades de discutir, ni tiene sentido hacerlo, si $A$ es en realidad un acto correcto o incorrecto. Por último, $\mathbf{R}_{2}$ no satisface el requisito mínimo que debe cumplir cualquier concepción normativa: servir de guia al comportamiento en situaciones dubitativas. La razón es simple. $\mathrm{Si}$ alguien pregunta " $\mathrm{Qué}$ harê?" cuando se enfrenta con un conflicto moral, difícilmente le sirve de algo que se le diga que debe hacer lo que piensa que debe hacer. Quien se encuentra en tal situación no sabe qué pensar y el precepto que proporciona $R_{2}$ no proporciona ningún tipo de ayuda en esa dirección (Hare [1960], 140-41). 
Estas observaciones criticas son suficientes para poner en duda la viabilidad de $R_{2}$. Cabe agregar a ellas que $R_{2}$ es incompatible, además, con el principio de universabilidad que muchos filósofos introducen para caracterizar uno de los rasgos típicos de los juicios con contenido moral. $\mathrm{N}$ es casual, pues, que Hare tome a $R_{2}$ como prototipo de posición relativista y que Frankena se refiera a $R_{2}$ con menosprecio (Frankena [1963], cap. 6). Dentro del relativismo normativo la variante generalmente asociada a $R_{1}$ no es $R_{2}$, sino una modificación de ella que surge al proyectar $\mathbf{R}_{2}$ al plano del grupo social. $\hat{\mathbf{R}}_{3}$-tal la denominaré- postula que si de conformidad con las pautas del grupo social al que pertenece un agente moral resulta incorrecto hacer $A$ en determinadas circunstancias, entonces es incorrecto para el agente moral hacer $A$ en tales circunstancias. Con otras palabras, la corrección (incorrección) de un acto con contenido moral depende de que el agente moral correspondiente se comporte de acuerdo a (en desacuerdo con) las pautas morales especificas que rigen en su grupo social. Existen varias críticas estándar contra $R_{3}$. La primera y principal es que, al igual que $R_{2}$, no se sigue de $R_{1}$ debido a que no es válido inferir pautas o normas a partir de enunciaciones descriptivas. Otra crítica importante es ésta: no resulta aceptable admitir sin reservas que el hecho de que el grupo social estipule qué debe hacerse en determinadas circunstancias pueda servir de justificación suficiente respecto de los cursos de comportamiento moral a adoptar, o adoptados. Una consecuencia inmediata de esto es que de aceptarse $R_{3}$ habría que tachar de inmorales al reformador social y al crítico de la moralidad establecida, pues los tipos de comportamientos que proponen, al apartarse de los marcos canónicos de comportamiento moral, resultan incorrectos. $Y$ este punto es demasiado fuerte para admitirlo sin más ni más. Resulta demasiado conservador; es decir, resulta llanamente obscurantista.

\section{Relativismo metaético}

Cuando se accede al nivel metaético (o nivel ético, en sentido estricto), esto es, al plano del estudio teórico-conceptual de la moralidad, las tesis relativistas muestran rasgos distntos a los que presentan $R_{1}, R_{2}$ y $R_{3}$. No pertenecen a este nivel tesis susceptibles de ser corroboradas o falsificadas empíricamente $\left(R_{1}\right)$, ni tampoco preceptos del tipo de $R_{2}$ y $R_{3}$. Las variantes relativistas que examinaré a continuación plantean problemas distintos a los planteados en los niveles anteriores.

La primera variante del relativismo metaético $\left(\mathbb{R}_{\underline{\xi}}\right.$ en adelante) consiste en sostener la validez de $R_{1}$ y aseverar que las opiniones contrapuestas acerca del mismo acto con contenido moral son igualmente válidas. Esta posición -que mencionara en II-, es sostenida con cierta predilección por antropólogos y sociólogos, quienes parecen a veces identificarla (erróneamente) con 
$R_{1}$ y otras veces parecen inferirla (erróneamente) de $R_{1}$. En realidad, $R_{4}$ posee un carácter distinto a $R_{1}$. Sin perjuicio de que, como ya he señalado, la aseveración de que las valoraciones morales contrapuestas acerca del mismo acto con contenido moral son igualmente válidas, tampoco se sigue de $R_{1}$.

La pregunta crucial que cabe hacer respecto de $\mathbf{R}_{4}$ es qué se entiende por "igualmente válidas" cuando se hace referencia a valoraciones morales contrapuestas. Hay un sentido de esta expresión, que no interesa mayormente. Es aquel según el cual decir que dos valoraciones morales contrapuestas son igualmente válidas significa que cada una de tales valoraciones es considerada correcta dentro del grupo social correspondiente. Este punto es, en realidad, una mera versión del corolario que sigue a $T_{1}$ y $T_{2}$, y corresponde al nivel empírico. El sentido de "igualmente válido" que interesa realmente en este contexto es más fuerte que el anterior y su elucidación permite imprimir un giro interesante a la discusión de $\mathbf{R}_{\mathbf{4}}$. En efecto, una respuesta posible a la pregunta acerca de qué se entiende por "igualmente válido" podría ser que cuando se afirma que dos valoraciones morales contrapuestas son igualmente válidas se quiere decir que las dos valoraciones poseen el mismo grado de plausibilidad dadas las condiciones en que se expresan. Es decir, que si se toman en cuenta tales condiciones no hay razón para preferir una valoración a la otra o para considerar que una es correcta mientras que la otra no lo es, etcétera. Sin embargo, esta respuesta dista de ser satisfactoria. Por un lado, es obvio que no permite adelantar mucho en la elucidación de "igualmente válido". Por el otro, muestra que los defensores de posiciones relativistas del tipo de $\mathbf{R}_{\mathbf{4}}$ juegan implícitamente con una presuposición importante. Supongamos que se señalara que $\mathbf{R}_{\mathbf{4}}$ es falsa porque es posible ofrecer un método, dar razones adecuadas $u$ ofrecer un criterio que con independencia de las opiniones de los agentes correspondientes y de su dependencia cultural, permite probar que una de las valoraciones morales en juego es, en si misma, correcta y que la otra no lo es. Es de suponer que el defensor de $\mathbf{R}_{4}$ negaria tal posibilidad. Pero en tal caso, cabría preguntar qué es lo que lo lleva a suponer que resulta imposible hallar un método, razones o criterios que permitan justificar una valoración moral en perjuicio de otra. Por cierto que el defensor de $\mathbf{R}_{4}$ no puede responder que tal creencia se funda en hallazgos empíricos, pues no se trata de $\mathbf{R}_{1}$ sino de una tesis específicamente filosófica. Tampoco tiene relevancia la supuesta explicación ofrecida antes acerca del significado de "igualmente válido". ¿En qué la fundamenta pues? La respuesta no puede hallarse de modo inmediato porque cuando se cuestiona en estos términos la viabilidad de $R_{4}$ sus defensores se mueven rápidamente en dirección a otra variante del relativismo, caracterizada por la afirmación explícita de que resulta imposible ofrecer una justificación racional de las valoraciones morales. Llamaré a esta nueva versión de relativismo, " $R_{5}$ ". El relativismo metodológico o el escepticismo ético, como se llama a veces a variantes de $\mathbf{R}_{5}$, 
sostiene básicamente que no existen en el campo de la moralidad criterios racionales que puedan ser empleados por seres humanos maduros para decidir, respecto de valoraciones morales contrapuestas, cuál es la correcta o la justificada, etcétera. Está claro que $\mathbf{R}_{\mathbf{5}}$ puede sostenerse sin apelar a ninguna de las variantes relativistas analizadas hasta el momento. El defensor de $\mathbf{R}_{5}$ no desconoce, por supuesto, que las teorias éticas ofrecen, casi sin excepción, criterios generales que intentan alcanzar el objetivo que $\mathbf{R}_{5}$ cancela. A tal efecto, las teorías éticas recurren a métodos de carácter cientifico (un rasgo típico de las teorías naturalistas), o a modalidades del conocimiento intuitivo (el método usual dentro del no naturalismo), o bien a formas sui generis (el caso de algunas versiones del no descriptivismo). El defensor de $R_{5}$ tampoco desconoce que en la vida moral cotidiana tanto él como sus semejantes apelan a razones que consideran buenas, para justificar cierto curso de acción o determinado juicio valorativo. Tampoco ignora que muchas veces hay consenso en cuanto a aceptar ciertas razones como buenas y a rechazar a otras por malas. Pero el defensor de $R_{5}$ no se satisface con ninguno de estos extremos. La pluralidad de doctrinas éticas lleva a preguntarse cuál de ellas es la verdadera y, paralelamente, cuál de los métodos de decisión postulados es, en realidad, el método racional. Por lo demás, si bien la vida cotidiana muestra casos de consenso, en el sentido indicado, también muestra muchísimos casos de disenso, con lo cual también este plano parece sugerir una posición como la que representa $R_{5}$.

Este tipo de argumentación es corriente entre los partidarios del relativismo ético. Su peso es grande por no concluyente. Puede argüirse al respeceto que si bien no existe de hecho un método racional unánimemente aceptado a nivel teórico no es diffcil concordar en cuanto a los requisitos que debe satisfacer y que, además, los modelos existentes brindan propuestas alternativas cuyo análisis y evaluación corresponde encarar como uno de los objetivos más importantes de la filosofía de la moral. El defensor de $\mathbf{R}_{5}$ puede argumentar en este punto que sin perjuicio del valor teórico de dichos modelos resulta imposible que alguno de ellos pueda llegar a satisfacer los requisitos exigidos. Pero es entonces cuando la posición del relativista ético pierde atractivo. En definitiva, todo ser racional -y el defensor de $R_{5}$ se supone que lo es- trata sistemáticamente de ser racional en el orden moral. Insistir en la búsqueda teórica del método en cuestión significa pues, entre otras cosas, proponerse explicar y clarificar las condiciones y el sentido del comportamiento racional en dicho orden. $\mathrm{Y}$ ésta es una tarea inexcusable para el filósofo de la moral. Con otras palabras, la defensa acérrima de $R_{5}$ parece comportar elementos contradictorios, no sólo con el comportamiento moral efectivo de sus sostenedores, sino también con una de las motivaciones teóricas más importantes en el desarrollo de la filosofía moral: explicar y clarificar el carácter racional del comportamiento moral. 


\section{Epilogo: relativismo y ciencias sociales}

¿Qué relevancia pueden tener estas consideraciones para la polémica acerca del compromiso valorativo de las ciencias sociales?

En primer lugar, resulta obvio que la introducción del compromiso valorativo en el campo cientifico tiene efectos inmediatos en la estabilidad de ciertos rasgos que hacen, tradicionalmente, a la esencia del conocimiento científico, a saber, corroboración intersubjetiva, neutralidad, racionalidad. Pero si estos rasgos entran de alguna manera en crisis es porque se sobreentiende que admitir el compromiso valorativo implica, ineludiblemente, aceptar algún tipo de posición relativista.

En segundo lugar, los supuestos relativistas no sólo son aceptados por la mayoría de los defensores de una concepción valorativamente comprometida de las ciencias sociales, sino que también son aceptados por muchos de los defensores de una concepción neutralista de las ciencias sociales. Adviértase que no es casual que una estrategia básica que siguen quienes sustentan esta última posición consiste en argumentar en favor de la posibilidad de neutralizar el marco valorativo, en distinguir tipos de juicios valorativos mostrando luego que algunos de ellos son susceptibles de verificación empírica, etcétera. La aceptación del relativismo no se limita, pues, a la clase de filósofos que sostienen el compromiso valorativo en las ciencias sociales. $\mathrm{Y}$ esto es importante tenerlo en cuenta.

Por último, el análisis desarrollado en II-V mueve a preguntarse hasta qué punto la polémica entablada no exige una elaboración detenida del tema del relativismo. Si lo expuesto hasta aquí tiene visos de admisibilidad -personalmente creo que lo tiene- la opción no sería entre el relativismo y el absolutismo sino más bien entre el relativismo, en cualquiera de las versiones analizadas, y lo que denominaré "no relativismo". Además, si las observaciones críticas formuladas en relación con cada uno de los tres tipos de relativismo son correctas, pareciera sensato decidir esta última opción en favor del no relativismo. Determinar qué efectos tendría en la polémica este cambio de frente es algo que excede los límites de este trabajo.

EDUardo A. RabossI

Consejo Nagional de Investigaciones

Científicas y Técnicas 


\section{BIBLIOGRAFIA}

S. E. Asch [1965], Social Psychology, Englewood Cliffs: Prentice-Hall.

R. Brandt [1959], Ethical Theory, Englewood Cliffs: Prentice-Hall.

R. M. Hare [1960], "Ethics" en J. O. Urmson (compilador), The Concise Encyclopedia of Philosophy and Philosophers, Londres: Hutchison.

E. Rabossi [1973], Notas sobre la moral y sus niveles metodológicos de estudio, La Plata: Instituto de Lógica y Filosofía de las Ciencias. 